\title{
Estrutura e florística de comunidade arbórea em duas áreas de Floresta Ombrófila Densa em Macaé, RJ
}

Tree community structure and floristics of two Ombrophilous Dense Forest areas at Macaé, Rio de Janeiro

\author{
Adriano Rosa Cruz ${ }^{1}$, Kelly Cristina da Silva-Gonçalves ${ }^{2,3}$ \& André Felippe Nunes-Freitas ${ }^{2,4}$
}

\begin{abstract}
Resumo
A Mata Atlântica cobria a maior parte do norte-fluminense, estando atualmente reduzida a menos de $10 \%$ de sua área original. Entretanto, pouco se sabe sobre a composição florística e a estrutura dos fragmentos florestais desta região. Este estudo visou caracterizar a composição florística e a estrutura de um trecho de Floresta Ombrófila Densa Submontana do Parque Natural Municipal Fazenda Atalaia (PNMFA - Macaé, RJ). Foram analisadas duas áreas com históricos de utilização distintos. Em cada área foram estabelecidas 30 parcelas de $100 \mathrm{~m}^{2}(10 \times 10 \mathrm{~m})$, onde os indivíduos arbóreos com DAP $\geq 5 \mathrm{~cm}$ foram registrados, identificados e mensurados. Foram amostrados 639 indivíduos de 118 espécies, dentro de 86 gêneros de 39 famílias botânicas. As famílias mais representativas foram Fabaceae, Euphorbiaceae, Myrtaceae, Meliaceae e Sapotaceae, com diferentes proporções de ocorrência entre as duas áreas analisadas. Nas duas áreas, Artocarpus heterophyllus Lam. (jaqueira) foi a espécie com maiores valores de todos os parâmetros fitossociológicos analisados. A área basal do PNMFA pode ser considerada de média a alta em relação a localidades próximas. Apesar da invasão por A. heterophyllus e as baixas densidades da maioria das espécies, as duas áreas parecem estar se recuperando e, com o manejo desta espécie exótica, é possível que a área volte a guardar uma parcela da diversidade biológica da região norte-fluminense.
\end{abstract}

Palavras-chave: floresta submontana, fitossociologia, região norte-fluminense, regeneração natural.

\begin{abstract}
The Atlantic Forest covered most of the Northern region of Rio de Janeiro state, being reduced, nowadays, to less than $10 \%$ of its original area. However, little is known about the floristic composition and structure of the forest fragments of this region. This study aimed to characterize the floristic composition and structure of a Dense Ombrophilous Submontane Forest at Parque Natural Municipal Fazenda Atalaia (PNMFA - Macaé, RJ). Two areas with different historical use were analyzed. In each area, 30 plots of $100 \mathrm{~m}^{2}(10 \times 10 \mathrm{~m})$ were established and the arboreal plants with $\mathrm{DBH}>5 \mathrm{~cm}$ were recorded and identified. A total of 639 individuals from 118 species, within 86 genera and 39 botanical families, were sampled. The most representative families were Fabaceae, Euphorbiaceae, Myrtaceae, Meliaceae and Sapotaceae, with different proportions of occurrence between the two areas analyzed. Artocarpus heterophyllus Lam. (jackfruit) had the highest values of all parameters in the phytosociological analysis. The basal area of PNMFA is considered medium to high compared to other locations. Despite the invasion by $A$. heterophyllus and low densities of most species, the two areas seem to be recovering, and with the management of this exotic species, it is possible that the area will preserve a portion of the biological diversity of northern Rio de Janeiro state.
\end{abstract}

Key words: submontane forest, phytosociology, Rio de Janeiro northern region, natural recovery.

\footnotetext{
${ }^{1}$ Centro Agronómico Tropical de Investigación y Enseñanza - CATIE, 3 km sobre carretera a Siquirres, C.P. 30501, Costa Rica.

${ }^{2}$ Universidade Federal Rural do Rio de Janeiro, Inst. Florestas, Programa de Pós-graduação em Ciências Ambientais e Florestais, Rodovia BR-465, km 7, 23890-000 Seropédica, RJ, Brasil

${ }^{3}$ Universidade Federal Rural do Rio de Janeiro, Inst. Florestas, Depto. Ciências Ambientais, Lab. Ecologia Florestal e Biologia Vegetal, Rodovia BR-465, km 7, 23890-000 Seropédica, RJ, Brasil

${ }^{4}$ Autor para correspondência: afnfreitas@ufrrj.br
} 


\section{Introdução}

A Mata Atlântica sensu lato (Oliveira-Filho \& Fontes 2000; Eisenlohr et al. 2011), segunda maior formação florestal brasileira, cobria originalmente uma área de 1,1 milhão de $\mathrm{km}^{2}$, o equivalente a cerca de $12 \%$ do território brasileiro (Morellato \& Haddad 2000; Oliveira-Filho \& Fontes 2000). No entanto, desde a década de 1980, a Mata Atlântica está reduzida a cerca de 7,5\% de sua área original, estando os seus remanescentes representados, em grande parte, por elevado número de fragmentos em diferentes graus de degradação, devido a diferentes atividades e pressões urbanas (Oliveira-Filho \& Fontes 2000; Fundação SOS Mata Atlântica \& INPE 2001; Rocha et al. 2003). Dessa forma, a Mata Atlântica apresenta-se como um mosaico de paisagens fragmentadas, formadas por um grande número de fragmentos florestais de pequeno porte (< 100 ha) e áreas em diversos estágios de degradação (Guatura et al. 1996), cercadas por matriz composta por diferentes tipos de cultivo, principalmente nas Regiões Sul e Sudeste (Oliveira-Filho \& Fontes 2000).

Assim como ocorreu em todo o Brasil, no Norte Fluminense a Mata Atlântica está reduzida a menos de $11 \%$ de sua área original (Carvalho 2001; Stehmann et al. 2009). As áreas de floresta até a altitude de $100 \mathrm{~m}$ (Floresta Ombrófila Densa de Terras Baixas e Submontana sensu Veloso et al. 1991) no passado foram substituídas por pastagens e plantações de cana-de-açúcar, estando a vegetação remanescente altamente fragmentada e distribuída, na sua maioria, em fragmentos com áreas inferiores a 1.000 ha, localizados, principalmente, em propriedades privadas (Carvalho 2001). Apesar do tamanho reduzido e relativo isolamento, boa parte destes remanescentes ainda apresenta elevada diversidade de espécies arbóreas (Pagano et al. 1987). Nesta região, o município de Macaé é um dos maiores, com 121.712 ha, dos quais 30.275 ha (24,9\%) são cobertos por fragmentos de florestas, onde há ocorrência de Floresta Ombrófila Densa de Terras Baixas e Submontana (Fundação SOS Mata Atlântica \& INPE 2010). No entanto, a facilidade de acesso às regiões de baixa declividade permitiu uma rápida e desordenada ocupação humana, levando a região a sofrer acelerada perda de suas áreas florestadas (Peres \& Rahy 2001).

Dentre os remanescentes de Mata Atlântica encontrados no município de Macaé, RJ, um dos que estão em melhor estado de conservação é o
Parque Natural Municipal da Fazenda Atalaia (PNMFA), que em 1995 foi transformado em unidade de conservação municipal. No entanto, pouco se conhece sobre a composição florística e a estrutura da vegetação deste fragmento, o que dificulta o estabelecimento de ações que visem ao manejo e à recuperação das áreas mais impactadas do Parque. Assim, este estudo teve com objetivo analisar a composição florística e a estrutura da vegetação arbórea de duas áreas do PNMFA e comparar sua composição, riqueza e diversidade com outras localidades da região norte do estado do Rio de Janeiro.

\section{Material e Métodos}

Área de estudo

O Parque Natural Municipal Fazenda Atalaia (PNMFA - 22 $15^{\prime}$ ' a $22^{\circ} 20^{\prime} \mathrm{S}$ e $42^{\circ} 58^{\prime}$ a $42^{\circ} 02^{\prime} \mathrm{W}$ ) está localizado no município de Macaé, estado do Rio de Janeiro, e possui cerca de $11 \mathrm{~km}$ de perímetro e uma área de 235 ha. A vegetação do PNMFA pode ser classificada como Floresta Ombrófila Densa de Terras Baixas e Submontana (Veloso et al. 1991), onde podem ser encontradas desde áreas de pasto abandonadas e vegetação secundária com diferentes idades de regeneração até trechos de mata que sofreram corte seletivo. O clima da região, segundo o sistema de Köppen, enquadra-se no macroclima $A_{w}$ (clima tropical com estação seca no inverno) (semi-úmido). A pluviosidade média anual é de $1.180 \mathrm{~mm}$, sendo dezembro o mês que apresenta os maiores valores de precipitação, com média de $180 \mathrm{~mm}$, enquanto agosto apresenta os menores valores, com média de $40 \mathrm{~mm}$ (SIMERJ 1990). A temperatura média anual é de $23,5^{\circ} \mathrm{C}$, sendo o mês mais quente janeiro, com máxima média de $26,9^{\circ} \mathrm{C}$. Já o mês mais frio é julho, com a temperatura mínima média de $19^{\circ} \mathrm{C}$ (SIMERJ 1990). A umidade relativa do ar média mensal em Macaé é praticamente constante ao longo do ano, variando entre 80 e $82 \%$ (SIMERJ 1990).

\section{Coleta e análise de dados}

Para analisar a estrutura e a diversidade florística do componente arbóreo do PNMFA, foram selecionadas duas áreas localizadas em altitudes e vertentes distintas do parque. A área 1 (A1) está localizada a cerca de $250 \mathrm{~m}$, na vertente Norte, e a área 2 (A2), a cerca de $120 \mathrm{~m}$, na vertente Sul. As duas áreas estão inseridas no 
vale do córrego do Ouro, onde existe, a cerca de $100 \mathrm{~m}$, uma pequena barragem que era usada no abastecimento de água da região. Estas áreas foram abandonadas há cerca de 50 anos, não sofrendo nenhum tipo de interferência antrópica desde então.

Em cada um das áreas, foram estabelecidas 30 parcelas de $100 \mathrm{~m}^{2}(10 \times 10 \mathrm{~m})$, em três linhas paralelas de amostragem, totalizando 60 parcelas e 0,6 ha, o equivalente a $0,26 \%$ da área do PNMFA. As linhas e as parcelas distavam $20 \mathrm{~m}$ uma das outras. Em cada parcela, foram amostrados todos os indivíduos com diâmetro à altura do peito (DAP a $1,30 \mathrm{~m}$ de altura do solo) $\geq 5,0 \mathrm{~cm}$. Todos os indivíduos que atenderam ao critério de inclusão tiveram DAP medido com trena diamétrica, sendo identificados em nível específico sempre que possível. O material testemunho das espécies amostradas foi coletado, identificado e encontra-se depositado na coleção de referência do Laboratório de Ecologia Florestal e Biologia Vegetal do Departamento de Ciências Ambientais/ UFRuralRJ. Foi utilizado o sistema de classificação APG III (APG III 2009) para a identificação das famílias botânicas.

Foram calculadas a riqueza de espécies (S) e o índice de diversidade de Shannon (H'), este último utilizando a base logarítmica neperiana (Magurran 2004) para o PNMFA como um todo e para cada uma das áreas amostradas. Para avaliar se os valores do índice de Shannon eram estatisticamente iguais, utilizou-se o teste $\mathrm{t}$ modificado de Hutchenson (Zar 1999). Foi gerada uma curva do coletor para avaliar o incremento da riqueza de espécies segundo o esforço amostral empregado e detectar se a curva atingia uma assíntota que representasse a eficiência do esforço amostral (Magurran 2004). Posteriormente, a curva do coletor obtida foi comparada com uma curva de rarefação gerada a partir de estimador Jackknife, utilizando-se um total de 1000 aleatorizações (Pillar 2004) por meio do programa EstimateS 8.2 (Cowell 2006). A composição de espécies das duas áreas amostradas foi comparada por meio de uma matriz de presença e ausência (Magurran 2004).

Para avaliar o grau de similaridade florística entre o PNMFA e outras áreas do Centro-Norte do estado foi utilizado o índice de Jaccard (Magurran 2004). Para avaliar o padrão de ordenação do PNMFA com outros estudos (Kurtz \& Araujo 2000; Silva \& Nascimento 2001; Borém \& OliveiraFilho 2002; Moreno et al. 2003; Rodrigues 2004; Carvalho et al. 2006a,b; Guedes-Bruni et al.
2006; Carvalho et al. 2007) (Tab. 1), foi utilizado o método escalonamento multidimensional nãométrico (NMDS), com auxílio do pacote estatístico Systat 12.0, (Wilkinson 1990) utilizando-se como medida de dissimilaridade a distância Bray-Curtis (Legendre \& Legendre 1998). Para esta análise foram excluídas da matriz de presença e ausência as espécies cuja identificação não estava em nível específico. A formação de possíveis grupos formados através do NMDS foi testada através de análise de similaridade (ANOSIM) (Clarke 1993).

Para caracterizar a estrutura da vegetação, foram calculados os parâmetros comumente empregados em trabalhos de levantamentos fitossociológicos: frequência absoluta (FA); frequência relativa (FR); densidade absoluta (DA); densidade relativa (DR); dominância absoluta (DoA); dominância relativa (DoR); valor de importância (VI); valor de cobertura (VC); e valor de importância (VI) (Mueller-Dombois \& Ellenberg 1974).

\section{Resultados}

Foram amostrados 639 indivíduos de 118 espécies, distribuídos em 86 gêneros de 39 famílias botânicas (Tabs. 1,2,3). Dos 639 indivíduos amostrados, um indivíduo foi identificado no nível de família e dois no nível de gênero, com todos os demais sendo identificados em nível específico (Tabs. 3,4). As famílias com os maiores valores de riqueza de espécies foram Fabaceae (13 espécies), Euphorbiaceae, Myrtaceae, Meliaceae (oito espécies cada) e Sapotaceae (sete espécies; Tabs. 2,3). Quando analisadas as duas áreas separadamente, foram amostrados 405 indivíduos em A 1 , pertencentes a 73 espécies de 56 gêneros e 28 famílias, enquanto em A2 foram encontrados 234 indivíduos de 88 espécies, 70 gêneros e 34 famílias (Tab. 2). As famílias Fabaceae (nove espécies), Myrtaceae (seis), Euphorbiaceae, Malvaceae e Meliaceae (cinco espécies cada) foram responsáveis por $41,1 \%$ do total de espécies amostradas em A1 (Tab. 2). Já em A2, as famílias mais representativas foram Myrtaceae (oito espécies), Fabaceae, Euphorbiaceae, Meliaceae (sete cada), Sapotaceae, Rubiaceae, Apocynaceae e Moraceae (cinco espécies cada), que representaram $55,7 \%$ do total de espécies (Tab. 2).

Quando comparadas com outras localidades do Norte do estado, a riqueza total do PNMFA (S $=118$ espécies) pode ser considerada intermediária (Tab. 1). O esforço amostral foi suficiente para 
Tabela 1 - Lista dos trabalhos utilizados na análise de similaridade florística. Número de indivíduos amostrados (N), número de famílias amostradas (F), riqueza de espécies $(\mathrm{S})$, diversidade biológica (H'), área amostrada (A), diâmetro a altura do peito (DAP) de inclusão, área basal $(\mathrm{AB})$ e método amostral utilizados nos estudos realizados na região centro-norte do estado do Rio de Janeiro.

Table 1 - List of studies used in the analysis of floristic similarity. Number of sampled individuals (N), number of sampled families (F), species richness (S), biological diversity index (H'), sampled area (A), breast height diameter of the inclusion criteria (DAP), basal area (AB) and sampling method used in studies conducted in localities of center-northern region of Rio de Janeiro state.

\begin{tabular}{|c|c|c|c|c|c|c|c|c|c|}
\hline Localidade & $\mathbf{N}$ & $\mathbf{F}$ & $\mathbf{S}$ & $\mathbf{H}^{\prime}$ & $\begin{array}{c}\text { A } \\
\text { (ha) }\end{array}$ & $\begin{array}{l}\text { DAP } \\
(\mathrm{cm})\end{array}$ & $\begin{array}{c}\mathrm{AB} \\
\left(\mathrm{m}^{2} / \mathrm{ha}\right)\end{array}$ & Método amostral & Autor \\
\hline PNMFA (Áreas 1 e 2) & 639 & 36 & 118 & 3,96 & 0,60 & 5,00 & 62,2 & 60 parcelas de $100 \mathrm{~m}^{2}$ & Presente estudo \\
\hline E.E.E. Paraíso & 592 & 42 & 83 & 4,20 & 2,50 & 5,00 & 57,28 & 150 pontos-quadrantes & Kurtz \& Araújo (2000) \\
\hline Mata do Carvão & 564 & 34 & 83 & 3,21 & 0,25 & 10,00 & 15,0 & 4 parcelas de $2500 \mathrm{~m}^{2}$ & Silva \& Nascimento (2001) \\
\hline Fazenda Biovert & 579 & 43 & 129 & 4,13 & 0,36 & & 23,15 & 6 parcelas de $600 \mathrm{~m}^{2}$ & Borém \& Oliveira-Filho (2002 \\
\hline Região do Imbé & 940 & 43 & 210 & 4,21 & 0,60 & 10,00 & 38,4 & 10 parcelas de $1300 \mathrm{~m}^{2}$ & Moreno et al. (2003) \\
\hline REBIO União & 882 & 44 & 250 & 4,90 & 1,20 & 10,00 & 32,0 & 12 parcelas de $1000 \mathrm{~m}^{2}$ & Rodrigues (2004) \\
\hline Região do Imbaú & 1598 & 42 & 161 & 3,26 & 0,20 & 5,00 & -- & 20 parcelas de $500 \mathrm{~m}^{2}$ & Carvalho et al. (2006a) \\
\hline Mata Rio Vermelho & 776 & 32 & 106 & 3,91 & 0,4 & 5,00 & 11,60 & 8 parcelas de $500 \mathrm{~m}^{2}$ & Carvalho et al. (2007) \\
\hline REBIO Poço das Antas & 580 & 45 & 174 & 4,57 & 1,00 & 10,00 & 23,77 & 40 parcelas de $250 \mathrm{~m}^{2}$ & Guedes-Bruni et al. (2006) \\
\hline Mata do Bom Jesus & 366 & 35 & 105 & 4,02 & - & 3,20 & 17,3 & 84 pontos-quadrantes & Carvalho et al. (2006b) \\
\hline
\end{tabular}

Tabela 2 - Riqueza de espécies (S) e proporção da riqueza de espécies (FR\%) de cada uma das famílias botânicas amostradas nas duas áreas estudadas no Parque Natural Municipal Fazenda Atalaia, município de Macaé, Rio de Janeiro. Table 2 - Species richness (S) and species richness proportion (FR\%) of each sampled plant families at two areas of Parque Natural Municipal Fazenda Atalaia, municipal district of Macaé, Rio de Janeiro.

\begin{tabular}{|c|c|c|c|c|c|c|}
\hline \multirow[b]{2}{*}{ Famílias } & \multicolumn{2}{|r|}{ A1 } & \multicolumn{2}{|r|}{ A2 } & \multicolumn{2}{|c|}{ Total } \\
\hline & $\mathbf{S}$ & FR $(\%)$ & $\mathbf{S}$ & FR $(\%)$ & $\mathbf{S}$ & FR $(\%)$ \\
\hline Anacardiaceae & 4 & 5,5 & 2 & 2,3 & 4 & 3,4 \\
\hline Annonaceae & 3 & 4,1 & 2 & 2,3 & 3 & 2,5 \\
\hline Apocynaceae & 2 & 2,7 & 5 & 5,7 & 5 & 4,2 \\
\hline Bignoniaceae & 2 & 2,7 & 2 & 2,3 & 3 & 2,5 \\
\hline Bombacaceae & 0 & 0 & 1 & 1,1 & 1 & 0,8 \\
\hline Boraginaceae & 1 & 1,4 & 0 & 0 & 1 & 0,8 \\
\hline Brassicaceae & 0 & 0 & 1 & 1,1 & 1 & 0,8 \\
\hline Burseraceae & 1 & 1,4 & 1 & 1,1 & 1 & 0,8 \\
\hline Cardiopteridaceae & 1 & 1,4 & 0 & 0 & 1 & 0,8 \\
\hline Celastraceae & 0 & 0 & 1 & 1,1 & 1 & 0,8 \\
\hline Chrysobalanaceae & 2 & 2,7 & 2 & 2,3 & 2 & 1,7 \\
\hline Elaeocarpaceae & 0 & 0 & 1 & 1,1 & 1 & 0,8 \\
\hline Euphorbiaceae & 5 & 6,8 & 7 & 8 & 8 & 6,8 \\
\hline Fabaceae & 9 & 12,3 & 7 & 8 & 13 & 11 \\
\hline Icacinaceae & 1 & 1,4 & 1 & 1,1 & 1 & 0,8 \\
\hline Lacistemaceae & 1 & 1,4 & 0 & 0 & 1 & 0,8 \\
\hline Lauraceae & 1 & 1,4 & 4 & 4,5 & 5 & 4,2 \\
\hline Lecythidaceae & 2 & 2,7 & 0 & 0 & 2 & 1,7 \\
\hline Malpighiaceae & 2 & 2,7 & 1 & 1,1 & 2 & 1,7 \\
\hline Malvaceae & 5 & 6,8 & 1 & 1,1 & 5 & 4,2 \\
\hline
\end{tabular}

\begin{tabular}{|c|c|c|c|c|c|c|}
\hline \multirow[b]{2}{*}{ Famílias } & \multicolumn{2}{|r|}{ A1 } & \multicolumn{2}{|r|}{$\mathbf{A 2}$} & \multicolumn{2}{|c|}{ Total } \\
\hline & $\mathbf{S}$ & $\operatorname{FR}(\%)$ & $\mathbf{S}$ & FR $(\%)$ & $\mathbf{S}$ & FR $(\%)$ \\
\hline Melastomataceae & 0 & 0 & 1 & 1,1 & 1 & 0,8 \\
\hline Meliaceae & 5 & 6,8 & 7 & 8 & 8 & 6,8 \\
\hline Monimiaceae & 2 & 2,7 & 3 & 3,4 & 3 & 2,5 \\
\hline Moraceae & 4 & 5,5 & 5 & 5,7 & 5 & 4,2 \\
\hline Myristicaceae & 1 & 1,4 & 0 & 0 & 1 & 0,8 \\
\hline Myrtaceae & 6 & 8,2 & 8 & 9,1 & 8 & 6,8 \\
\hline Nyctaginaceae & 1 & 1,4 & 1 & 1,1 & 1 & 0,8 \\
\hline Ochnaceae & 0 & 0 & 1 & 1,1 & 1 & 0,8 \\
\hline Phyllantaceae & 0 & 0 & 1 & 1,1 & 1 & 0,8 \\
\hline Phytolaccaceae & 0 & 0 & 1 & 1,1 & 2 & 1,7 \\
\hline Polygalaceae & 0 & 0 & 1 & 1,1 & 1 & 0,8 \\
\hline Rubiaceae & 2 & 2,7 & 5 & 5,7 & 5 & 4,2 \\
\hline Rutaceae & 0 & 0 & 2 & 2,3 & 2 & 1,7 \\
\hline Sapindaceae & 3 & 4,1 & 3 & 3,4 & 5 & 4,2 \\
\hline Sapotaceae & 3 & 4,1 & 5 & 5,7 & 7 & 5,9 \\
\hline Siparunaceae & 1 & 1,4 & 1 & 1,1 & 1 & 0,8 \\
\hline Solanaceae & 2 & 2,7 & 1 & 1,1 & 2 & 1,7 \\
\hline Urticaceae & 1 & 1,4 & 2 & 2,3 & 2 & 1,7 \\
\hline Vochysiaceae & 0 & 0 & 1 & 1,1 & 1 & 0,8 \\
\hline RIQUEZA & 73 & 100 & 88 & 100 & 118 & 100 \\
\hline
\end{tabular}


Tabela 3 - Parâmetros fitossociológicos das espécies amostradas na Área 1 (A1), no Parque Natural Municipal Fazenda Atalaia, município de Macaé, Rio de Janeiro, ordenadas de forma decrescente por Valor de Importância (VI). $\mathrm{FA}=$ frequência absoluta; $\mathrm{FR}=$ frequência relativa (\%); DA = densidade absoluta (indivíduos/ha); DR = densidade relativa (\%); DoA = dominância absoluta $\left(\mathrm{m}^{2} / \mathrm{ha}\right)$; DoR = dominância relativa $(\%)$; VC = valor de cobertura.

Table 3 - Phytosociological parameters of the sampled species at Area 1 (A1), at Parque Natural Municipal Fazenda Atalaia, municipal district of Macaé, Rio de Janeiro, ordered by Importance Value (VI). FA = absolute frequency; FR = relative frequency (\%); DA = absolute density (individuals/ha); $\mathrm{DR}=$ relative density $(\%) ; \mathrm{DoA}=$ absolute dominance $\left(\mathrm{m}^{2} / \mathrm{ha}\right) ; \mathrm{DoR}=$ relative dominance $(\%) ; \mathrm{VC}=$ cover value.

\begin{tabular}{|c|c|c|c|c|c|c|c|c|c|c|}
\hline Família & Nome Científico & FA & FR & DA & DR & DoA & DoR & $\mathrm{VC}$ & VI & $\begin{array}{c}\mathrm{N}^{\mathbf{o}} \\
\text { coletor }\end{array}$ \\
\hline Moraceae & Artocarpus heterophyllus Lam. & 3 & 10 & 213 & 15,8 & 13,26 & 37,25 & 53,1 & 63,1 & $\mathrm{~s} / \mathrm{n}^{\mathrm{o}}$ \\
\hline Nyctaginaceae & Guapira opposita (Vell.) Reitz & 3 & 10 & 173 & 12,8 & 0,50 & 1,39 & 14,2 & 24,2 & ARC 46 \\
\hline Euphorbiaceae & Senefeldera verticilata (Vell.) Croizat & 3 & 10 & 90 & 6,7 & 1,10 & 3,10 & 9,8 & 19,8 & ARC 58 \\
\hline Euphorbiaceae & Croton floribundus Spreng & 3 & 10 & 67 & 4,9 & 1,25 & 3,50 & 8,4 & 18,4 & ARC 16 \\
\hline Myrtaceae & Eugenia tinguyensis Cambess. & 4 & 13,3 & 33 & 2,5 & 0,24 & 0,67 & 3,1 & 16,5 & ARC 17 \\
\hline Sapindaceae & Cupania vernalis Cambess. & 3 & 10 & 43 & 3,2 & 0,85 & 2,39 & 5,6 & 15,6 & ARC 18 \\
\hline Meliaceae & Guarea macrophylla (Vell.) T.D. Penn & 2 & 6,7 & 37 & 2,7 & 1,30 & 3,67 & 6,4 & 13 & ARC 35 \\
\hline Moraceae & Ficus adhatodifolia Schott & 1 & 3,3 & 10 & 0,7 & 2,94 & 8,26 & 9 & 12,3 & ARC 38 \\
\hline Annonaceae & Oxandra nitida R.E. Fries & 3 & 10 & 20 & 1,5 & 0,03 & 0,09 & 1,6 & 11,6 & ARC 11 \\
\hline Lauraceae & Ocotea divaricata (Nees) Mez & 2 & 6,7 & 37 & 2,7 & 0,53 & 1,48 & 4,2 & 10,9 & $\mathrm{~s} / \mathrm{n}^{\mathrm{o}}$ \\
\hline Urticaceae & Cecropia pachystachya Trécul & 2 & 6,7 & 17 & 1,2 & 1,03 & 2,89 & 4,1 & 10,8 & $\mathrm{~s} / \mathrm{n}^{\mathrm{o}}$ \\
\hline Sapindaceae & Cupania oblongifolia Mart & 2 & 6,7 & 23 & 1,7 & 0,58 & 1,62 & 3,3 & 10 & ARC 16 \\
\hline Siparunaceae & Siparuna guianensis Aubl. & 2 & 6,7 & 33 & 2,5 & 0,10 & 0,27 & 2,7 & 9,4 & ARC 02 \\
\hline Myristicaceae & Virola bicuhyba (Schott) Warb. & 2 & 6,7 & 23 & 1,7 & 0,19 & 0,54 & 2,3 & 8,9 & ARC 03 \\
\hline Fab. Caesalpinioideae & Apuleia leiocarpa (Vogel) J. F. Macbr. & 2 & 6,7 & 10 & 0,7 & 0,47 & 1,33 & 2,1 & 8,7 & ARC 07 \\
\hline Bignoniaceae & Cybistax antisyphilitica (Mart.) Mart. & 1 & 3,3 & 20 & 1,5 & 1,35 & 3,80 & 5,3 & 8,6 & ARC 45 \\
\hline Myrtaceae & Myrcia splendens (Sw.) DC. & 2 & 6,7 & 13 & 1 & 0,30 & 0,84 & 1,8 & 8,5 & ARC 01 \\
\hline Sapotaceae & Pouteria bangii (Rusby) T.P. Penn. & 2 & 6,7 & 23 & 1,7 & 0,03 & 0,09 & 1,8 & 8,5 & ARC 36 \\
\hline Rubiaceae & Genipa americana $\mathrm{L}$. & 2 & 6,7 & 20 & 1,5 & 0,12 & 0,32 & 1,8 & 8,5 & ARC 37 \\
\hline Fab. Mimosoideae & Plathymenia reticulata Benth. & 1 & 3,3 & 20 & 1,5 & 1,23 & 3,47 & 4,9 & 8,3 & ARC 04 \\
\hline Malvaceae & Pterigota brasiliensis Fr. All. & 2 & 6,7 & 10 & 0,7 & 0,29 & 0,81 & 1,5 & 8,2 & ARC 05 \\
\hline Myrtaceae & Calyptranthes concinna DC. & 2 & 6,7 & 17 & 1,2 & 0,11 & 0,30 & 1,5 & 8,2 & ARC 34 \\
\hline Myrtaceae & Campomanesia laurifolia Gardner & 2 & 6,7 & 17 & 1,2 & 0,04 & 0,11 & 1,3 & 8 & ARC 12 \\
\hline Solanaceae & Solanum swartzianum Roem. \& Schult & 2 & 6,7 & 17 & 1,2 & 0,04 & 0,11 & 1,3 & 8 & ARC 13 \\
\hline Meliaceae & Trichilia elegans A. Juss & 2 & 6,7 & 17 & 1,2 & 0,02 & 0,05 & 1,3 & 7,9 & ARC 22 \\
\hline Meliaceae & Trichilia lepidota Mart. & 2 & 6,7 & 10 & 0,7 & 0,16 & 0,45 & 1,2 & 7,9 & ARC 07 \\
\hline Anacardiaceae & Astronium graveolens Jacq. & 2 & 6,7 & 13 & 1 & 0,05 & 0,15 & 1,1 & 7,8 & ARC 06 \\
\hline Cardiopteridaceae & Citronella paniculata (Mart.)R.A. Howard & 2 & 6,7 & 10 & 0,7 & 0,02 & 0,06 & 0,8 & 7,5 & ARC 10 \\
\hline Anacardiaceae & Astronium fraxifolium Schott. Ex Spreng. & 1 & 3,3 & 3 & 0,2 & 1,34 & 3,77 & 4 & 7,4 & ARC 49 \\
\hline Solanaceae & Solanum granulosoleprosum Dunal & 1 & 3,3 & 3 & 0,2 & 1,25 & 3,51 & 3,8 & 7,1 & ARC 55 \\
\hline
\end{tabular}




\begin{tabular}{|c|c|c|c|c|c|c|c|c|c|c|}
\hline Família & Nome Científico & FA & FR & DA & DR & DoA & DoR & VC & VI & $\begin{array}{c}\mathrm{N}^{\mathbf{o}} \\
\text { coletor }\end{array}$ \\
\hline Apocynaceae & Geissospermum laeve (Vell.) Miers & 1 & 3,3 & 10 & 0,7 & 0,49 & 1,38 & 2,1 & 5,5 & ARC 61 \\
\hline Rubiaceae & Psychotria nuda (Cham. \& Schltdl.) Wawra & 1 & 3,3 & 10 & 0,7 & 0,41 & 1,16 & 1,9 & 5,2 & $\mathrm{~s} / \mathrm{n}^{\mathrm{o}}$ \\
\hline Malvaceae & Sterculia chicha A.St.-Hil. & 1 & 3,3 & 10 & 0,7 & 0,41 & 1,16 & 1,9 & 5,2 & ARC 62 \\
\hline Moraceae & Ficus gomelleira Kunth \& C.D. Bouché & 1 & 3,3 & 7 & 0,5 & 0,49 & 1,38 & 1,9 & 5,2 & ARC 60 \\
\hline Burseraceae & Protium widgrenii Engl. & 1 & 3,3 & 20 & 1,5 & 0,11 & 0,32 & 1,8 & 5,1 & ARC 50 \\
\hline Sapotaceae & Diploon sp. & 1 & 3,3 & 10 & 0,7 & 0,36 & 1 & 1,7 & 5,1 & ARC 51 \\
\hline Apocynaceae & Aspidosperma ramiflorum Müll. Arg. & 1 & 3,3 & 10 & 0,7 & 0,24 & 0,67 & 1,4 & 4,7 & ARC 08 \\
\hline Euphorbiaceae & Alchornea triplinervia (Spreng.) Müll. Arg. & 1 & 3,3 & 7 & 0,5 & 0,32 & 0,90 & 1,4 & 4,7 & ARC 09 \\
\hline Malvaceae & Luehea divaricata Mart. & 1 & 3,3 & 10 & 0,7 & 0,23 & 0,64 & 1,4 & 4,7 & ARC 14 \\
\hline Annonaceae & Annona cacans Warm. & 1 & 3,3 & 7 & 0,5 & 0,25 & 0,70 & 1,2 & 4,5 & $\mathrm{~s} / \mathrm{n}^{\mathrm{o}}$ \\
\hline Monimiaceae & Mollinedia heteranthera Perkins & 1 & 3,3 & 13 & 1 & 0,02 & 0,07 & 1,1 & 4,4 & ARC 19 \\
\hline Myrtaceae & Marlierea tomentosa Cambess. & 1 & 3,3 & 10 & 0,7 & 0,10 & 0,27 & 1 & 4,3 & ARC 20 \\
\hline Myrtaceae & Myrcia plusiantha Kiaersk & 1 & 3,3 & 10 & 0,7 & 0,10 & 0,27 & 1 & 4,3 & ARC 27 \\
\hline Moraceae & Brosimum guianense (Aubl.) Huber & 1 & 3,3 & 7 & 0,5 & 0,16 & 0,45 & 0,9 & 4,3 & ARC 28 \\
\hline Bignoniaceae & Tabebuia cassinoides (Lam.) DC. & 1 & 3,3 & 10 & 0,7 & 0,07 & 0,18 & 0,9 & 4,3 & ARC 33 \\
\hline Chrysobalanaceae & Licania tomentosa (Benth.) Fritsch & 1 & 3,3 & 10 & 0,7 & 0,05 & 0,15 & 0,9 & 4,2 & ARC 32 \\
\hline Sapotaceae & Diploon cuspidatum (Hoehne) Cronquist & 1 & 3,3 & 10 & 0,7 & 0,05 & 0,13 & 0,9 & 4,2 & ARC 39 \\
\hline Fab. Papilionoideae & Lonchocarpus filipes Benth & 1 & 3,3 & 10 & 0,7 & 0,04 & 0,10 & 0,8 & 4,2 & ARC 41 \\
\hline Euphorbiaceae & Alchornea glandulosa Poepp. & 1 & 3,3 & 3 & 0,2 & 0,21 & 0,58 & 0,8 & 4,2 & ARC 42 \\
\hline Lecythidaceae & Cariniana legalis (Mart.) Kuntze & 1 & 3,3 & 10 & 0,7 & 0,02 & 0,06 & 0,8 & 4,1 & ARC 49 \\
\hline Lecythidaceae & Cariniana estrellensis (Raddi) Kuntze & 1 & 3,3 & 10 & 0,7 & 0,02 & 0,06 & 0,8 & 4,1 & ARC 72 \\
\hline Boraginaceae & Cordia superba Cham. & 1 & 3,3 & 10 & 0,7 & 0,02 & 0,05 & 0,8 & 4,1 & ARC 73 \\
\hline Chrysobalanaceae & Licania kunthiana Hook. F. & 1 & 3,3 & 7 & 0,5 & 0,10 & 0,28 & 0,8 & 4,1 & ARC 88 \\
\hline Fabaceae & Fabaceae & 1 & 3,3 & 3 & 0,2 & 0,14 & 0,40 & 0,6 & 4 & $\mathrm{~s} / \mathrm{n}^{\mathrm{o}}$ \\
\hline Malvaceae & Guazuma ulmifolia Lam. & 1 & 3,3 & 7 & 0,5 & 0,03 & 0,10 & 0,6 & 3,9 & ARC 70 \\
\hline Fab. Papilionoideae & Lonchocarpus cultratus (Vell.) A.M.G. & 1 & 3,3 & 7 & 0,5 & 0,03 & 0,09 & 0,6 & 3,9 & ARC 69 \\
\hline Anacardiaceae & Tapirira guianensis Aubl. & 1 & 3,3 & 7 & 0,5 & 0,03 & 0,09 & 0,6 & 3,9 & $\mathrm{~s} / \mathrm{n}^{\mathrm{o}}$ \\
\hline Monimiaceae & Mollinedia oligantha Perkins & 1 & 3,3 & 7 & 0,5 & 0,02 & 0,05 & 0,5 & 3,9 & ARC 31 \\
\hline Euphorbiaceae & Croton urucurana Baill. & 1 & 3,3 & 3 & 0,2 & 0,11 & 0,30 & 0,5 & 3,9 & ARC 52 \\
\hline Lacistemaceae & Lacistema pubescens Mart. & 1 & 3,3 & 7 & 0,5 & 0,01 & 0,04 & 0,5 & 3,9 & ARC 53 \\
\hline Malpighiaceae & Byrsonima sericea DC. & 1 & 3,3 & 7 & 0,5 & 0,01 & 0,03 & 0,5 & 3,9 & ARC 54 \\
\hline Malvaceae & Pseudobombax grandiflorum (Cav.) A.Robyns & 1 & 3,3 & 3 & 0,2 & 0,04 & 0,13 & 0,4 & 3,7 & ARC 63 \\
\hline Icacinaceae & Leretia cordata Vel. & 1 & 3,3 & 3 & 0,2 & 0,04 & 0,10 & 0,3 & 3,7 & ARC 64 \\
\hline Fab. Papilionoideae & Machaerium nictitans (Vell.) Benth. & 1 & 3,3 & 3 & 0,2 & 0,03 & 0,09 & 0,3 & 3,7 & ARC 65 \\
\hline Fab. Mimosoideae & Piptadenia gonoacantha (Mart.) J. F. Macbr. & 1 & 3,3 & 3 & 0,2 & 0,03 & 0,08 & 0,3 & 3,7 & $\mathrm{~s} / \mathrm{n}^{\circ}$ \\
\hline
\end{tabular}




\begin{tabular}{|c|c|c|c|c|c|c|c|c|c|c|}
\hline Família & Nome Científico & FA & FR & DA & DR & DoA & DoR & $\mathrm{VC}$ & VI & $\begin{array}{c}\mathrm{N}^{\circ} \\
\text { coletor }\end{array}$ \\
\hline Meliaceae & Trichilia casaretti C. DC. & 1 & 3,3 & 3 & 0,2 & 0,03 & 0,07 & 0,3 & 3,7 & $\mathrm{~s} / \mathrm{n}^{\mathrm{o}}$ \\
\hline Fab. Mimosoideae & Peltophorum dubium (Sprengel) Taub & 1 & 3,3 & 3 & 0,2 & 0,02 & 0,05 & 0,3 & 3,6 & $\mathrm{~s} / \mathrm{n}^{\mathrm{o}}$ \\
\hline Anacardiaceae & Spondias mombin L. & 1 & 3,3 & 3 & 0,2 & 0,01 & 0,03 & 0,3 & 3,6 & ARC 71 \\
\hline Meliaceae & Trichilia pseudostipularis (A.Juss.) C.DC. & 1 & 3,3 & 3 & 0,2 & 0,01 & 0,03 & 0,3 & 3,6 & ARC 99 \\
\hline Malpighiaceae & $\begin{array}{l}\text { Barnebya dispar (Griseb.) W.R.Anderson } \\
\& \text { B.Gates }\end{array}$ & 1 & 3,3 & 3 & 0,2 & 0,01 & 0,03 & 0,3 & 3,6 & ARC 106 \\
\hline Annonaceae & Oxandra martiana (Schltdl.) R.E. Fr. & 1 & 3,3 & 3 & 0,2 & 0,01 & 0,02 & 0,3 & 3,6 & ARC 110 \\
\hline Sapindaceae & Tripterodendron filicifolium Radlk & 1 & 3,3 & 3 & 0,2 & 0,01 & 0,02 & 0,3 & 3,6 & ARC 88 \\
\hline \multirow[t]{2}{*}{ Fab. Papilionoideae } & Myrocarpus frondosus Allemão & 1 & 3,3 & 3 & 0,2 & 0,01 & 0,02 & 0,3 & 3,6 & ARC 101 \\
\hline & TOTAIS & & & 1350 & 100 & 35,60 & 100 & 200 & 553,3 & \\
\hline
\end{tabular}

$\mathrm{ARC}=$ Adriano Rosa Cruz

Tabela 4 - Parâmetros fitossociológicos das espécies amostradas na Área 2 (A2), no Parque Natural Municipal Fazenda Atalaia, município de Macaé, Rio de Janeiro, ordenadas de forma decrescente por Valor de Importância (VI). FA = frequência absoluta; $\mathrm{FR}=$ frequência relativa (\%); $\mathrm{DA}=$ densidade absoluta (indivíduos/ha); $\mathrm{DR}=$ densidade relativa (\%); DoA = dominância absoluta ( $\left.\mathrm{m}^{2} / \mathrm{ha}\right)$; DoR = dominância relativa (\%); $\mathrm{VC}=$ valor de cobertura.

Table 4 - Phytosociological parameters of the sampled species at Area 2 (A2), at Parque Natural Municipal Fazenda Atalaia, municipal district of Macaé, Rio de Janeiro, ordered by Importance Value (VI). FA = absolute frequency FR = relative frequency $(\%)$; DA = absolute density (individuals/ha); $\mathrm{DR}=$ relative density $(\%) ; \mathrm{DoA}=$ absolute dominance $\left(\mathrm{m}^{2} / \mathrm{ha}\right) ; \mathrm{DoR}=$ relative dominance $(\%) ; \mathrm{VC}=$ cover value.

\begin{tabular}{|c|c|c|c|c|c|c|c|c|c|c|}
\hline Família & Nome Científico & FA & FR & $\mathbf{D A}$ & DR & DoA & DoR & $\mathrm{VC}$ & VI & $\begin{array}{c}\mathrm{N}^{0} \\
\text { coletor }\end{array}$ \\
\hline Moraceae & Artocarpus heterophyllus Lam. & 3 & 10 & 70 & 9 & 4,86 & 18,27 & 27,2 & 37,2 & $\mathrm{~s} / \mathrm{n}^{\mathrm{o}}$ \\
\hline Moraceae & Ficus gomelleira Kunth \& C.D. Bouché & 2 & 6,7 & 10 & 1,3 & 4,94 & 18,56 & 19,8 & 26,5 & ARC 60 \\
\hline Euphorbiaceae & Croton floribundus Spreng & 2 & 6,7 & 40 & 5,1 & 1,43 & 5,36 & 10,5 & 17,2 & ARC 16 \\
\hline Meliaceae & Guarea macrophylla (Vell.) T.D. Penn & 3 & 10 & 37 & 4,7 & 0,63 & 2,37 & 7,1 & 17,1 & ARC 35 \\
\hline Moraceae & Brosimum guianense (Aubl.) Huber & 2 & 6,7 & 47 & 6 & 0,86 & 3,24 & 9,2 & 15,9 & ARC 28 \\
\hline Meliaceae & Cedrella fissilis Vell. & 2 & 6,7 & 7 & 0,9 & 2,12 & 7,98 & 8,8 & 15,5 & ARC 123 \\
\hline Euphorbiaceae & Senefeldera verticilata (Vell.) Croizat & 3 & 10 & 33 & 4,3 & 0,30 & 1,12 & 5,4 & 15,4 & ARC 58 \\
\hline Sapindaceae & Cupania oblongifolia Mart & 3 & 10 & 27 & 3,4 & 0,40 & 1,52 & 4,9 & 14,9 & ARC 16 \\
\hline Nyctaginaceae & Guapira opposita (Vell.) Reitz & 2 & 6,7 & 50 & 6,4 & 0,37 & 1,40 & 7,8 & 14,5 & ARC 46 \\
\hline Rubiaceae & Genipa americana $\mathrm{L}$. & 2 & 6,7 & 37 & 4,7 & 0,39 & 1,48 & 6,2 & 12,8 & ARC 37 \\
\hline Euphorbiaceae & Croton urucurana Baill. & 2 & 6,7 & 13 & 1,7 & 0,39 & 1,45 & 3,2 & 9,8 & ARC 52 \\
\hline Euphorbiaceae & Mabea fistulifera Mart. & 2 & 6,7 & 13 & 1,7 & 0,31 & 1,17 & 2,9 & 9,5 & ARC 119 \\
\hline Myrtaceae & Eugenia umbrosa O. Berg. & 2 & 6,7 & 13 & 1,7 & 0,19 & 0,70 & 2,4 & 9,1 & ARC 133 \\
\hline Sapotaceae & Diploon cuspidatum (Hoehne) Cronquist & 2 & 6,7 & 10 & 1,3 & 0,20 & 0,74 & 2 & 8,7 & ARC 39 \\
\hline Siparunaceae & Siparuna guianensis Aubl. & 2 & 6,7 & 13 & 1,7 & 0,06 & 0,23 & 1,9 & 8,6 & ARC 32 \\
\hline Rubiaceae & $\begin{array}{l}\text { Psychotria nuda (Cham. \& Schltdl.) } \\
\text { Wawra }\end{array}$ & 2 & 6,7 & 10 & 1,3 & 0,14 & 0,54 & 1,8 & 8,5 & $\mathrm{~s} / \mathrm{n}^{\circ}$ \\
\hline Monimiaceae & Mollinedia heteranthera Perkins & 2 & 6,7 & 7 & 0,9 & 0,09 & 0,33 & 1,2 & 7,8 & ARC 19 \\
\hline Fab. Mimosoideae & Inga vera Wild. & 2 & 6,7 & 7 & 0,9 & 0,06 & 0,24 & 1,1 & 7,8 & ARC 135 \\
\hline
\end{tabular}




\begin{tabular}{|c|c|c|c|c|c|c|c|c|c|c|}
\hline Família & Nome Científico & FA & FR & DA & DR & DoA & DoR & VC & VI & $\begin{array}{c}N^{o} \\
\text { coletor } \\
\end{array}$ \\
\hline Apocynaceae & Malouetia arborea (Vell.) Miers & 2 & 6,7 & 7 & 0,9 & 0,06 & 0,23 & 1,1 & 7,7 & $\overline{\mathrm{ARC} 134}$ \\
\hline Myrtaceae & Eugenia tinguyensis Cambess. & 2 & 6,7 & 7 & 0,9 & 0,05 & 0,18 & 1 & 7,7 & ARC 17 \\
\hline Rutaceae & Zanthoxylum riedelianum Engl. & 2 & 6,7 & 7 & 0,9 & 0,04 & 0,16 & 1 & 7,7 & ARC 138 \\
\hline Annonaceae & Guatteria australis A.St.-Hil. & 2 & 6,7 & 7 & 0,9 & 0,03 & 0,11 & 1 & 7,6 & ARC 140 \\
\hline Sapotaceae & Manilkara longifolia (A.DC.) Dubard & 2 & 6,7 & 7 & 0,9 & 0,02 & 0,07 & 0,9 & 7,6 & ARC 139 \\
\hline Polygonaceae & Coccoloba alnifolia Casar. & 1 & 3,3 & 13 & 1,7 & 0,54 & 2,04 & 3,8 & 7,1 & ARC 177 \\
\hline Fab. Caesalpinioideae & Apuleia leiocarpa (Vogel) J. F. Macbr. & 1 & 3,3 & 3 & 0,4 & 0,86 & 3,23 & 3,7 & 7 & ARC 07 \\
\hline Malvaceae & $\begin{array}{l}\text { Pseudobombax grandiflorum (Cav.) } \\
\text { A.Robyns }\end{array}$ & 1 & 3,3 & 10 & 1,3 & 0,53 & 1,99 & 3,3 & 6,6 & ARC 63 \\
\hline Icacinaceae & Leretia cordata Vell. & 1 & 3,3 & 3 & 0,4 & 0,72 & 2,71 & 3,1 & 6,5 & ARC 64 \\
\hline Rubiaceae & Psychotria vellosiana Benth & 1 & 3,3 & 3 & 0,4 & 0,56 & 2,10 & 2,5 & 5,9 & $\mathrm{~s} / \mathrm{n}^{\circ}$ \\
\hline Anacardiaceae & Astronium graveolens Jacq. & 1 & 3,3 & 7 & 0,9 & 0,43 & 1,62 & 2,5 & 5,8 & ARC 06 \\
\hline Urticaceae & Cecropia pachystachya Trécul & 1 & 3,3 & 7 & 0,9 & 0,30 & 1,14 & 2 & 5,3 & $\mathrm{~s} / \mathrm{n}^{\circ}$ \\
\hline Fab. Mimosoideae & Anadenanthera peregrina (L.) Speg. & 1 & 3,3 & 3 & 0,4 & 0,39 & 1,48 & 1,9 & 5,2 & ARC 178 \\
\hline Ochnaceae & Ouratea parviflora Baill Tiegh. & 1 & 3,3 & 13 & 1,7 & 0,02 & 0,08 & 1,8 & 5,1 & ARC 188 \\
\hline Monimiaceae & $\begin{array}{l}\text { Mollinedia puberula (Puberula J.R. } \\
\text { Perkins) }\end{array}$ & 1 & 3,3 & 13 & 1,7 & 0,01 & 0,05 & 1,8 & 5,1 & $\mathrm{~s} / \mathrm{n}^{\circ}$ \\
\hline Sapotaceae & Ecclinusa ramiflora Mart. & 1 & 3,3 & 7 & 0,9 & 0,24 & 0,89 & 1,7 & 5,1 & ARC 198 \\
\hline Fab. Caesalpinioideae & Peltophorum dubium (Sprengel) Taub & 1 & 3,3 & 7 & 0,9 & 0,21 & 0,79 & 1,6 & 5 & $\mathrm{~s} / \mathrm{n}^{\circ}$ \\
\hline Rubiaceae & Bathysa cf. mendoncaei K. Schum. & 1 & 3,3 & 10 & 1,3 & 0,09 & 0,35 & 1,6 & 5 & ARC 189 \\
\hline Euphorbiaceae & Alchornea glandulosa Poepp. & 1 & 3,3 & 3 & 0,4 & 0,28 & 1,06 & 1,5 & 4,8 & ARC 42 \\
\hline Rutaceae & Zanthoxylum rhoifolium Engl. & 1 & 3,3 & 10 & 1,3 & 0,05 & 0,20 & 1,5 & 4,8 & ARC 136 \\
\hline Burseraceae & Protium widgrenii Engl. & 1 & 3,3 & 7 & 0,9 & 0,16 & 0,62 & 1,5 & 4,8 & ARC 51 \\
\hline Annonaceae & Annona cacans Warm. & 1 & 3,3 & 3 & 0,4 & 0,24 & 0,92 & 1,3 & 4,7 & $\mathrm{~s} / \mathrm{n}^{\circ}$ \\
\hline Meliaceae & Cedrela odorata L. & 1 & 3,3 & 3 & 0,4 & 0,23 & 0,88 & 1,3 & 4,6 & ARC 137 \\
\hline Sapindaceae & Matayba guianensis Aubl. & 1 & 3,3 & 3 & 0,4 & 0,23 & 0,88 & 1,3 & 4,6 & $\mathrm{~s} / \mathrm{n}^{\circ}$ \\
\hline Vochysiaceae & Vochysia oppugnata (Vell.) Warm. & 1 & 3,3 & 7 & 0,9 & 0,11 & 0,40 & 1,3 & 4,6 & $\mathrm{~s} / \mathrm{n}^{\circ}$ \\
\hline Moraceae & Ficus adhatodifolia Schott & 1 & 3,3 & 7 & 0,9 & 0,09 & 0,34 & 1,2 & 4,5 & ARC 38 \\
\hline Sapindaceae & Cupania vernalis Cambess. & 1 & 3,3 & 7 & 0,9 & 0,08 & 0,30 & 1,2 & 4,5 & ARC 18 \\
\hline Monimiaceae & Mollinedia oligantha Perkins & 1 & 3,3 & 7 & 0,9 & 0,06 & 0,24 & 1,1 & 4,4 & ARC 31 \\
\hline Bombacaceae & $\begin{array}{l}\text { Eriotheca candolleana (K.Schum.) } \\
\text { A.Robyns }\end{array}$ & 1 & 3,3 & 3 & 0,4 & 0,17 & 0,65 & 1,1 & 4,4 & ARC 199 \\
\hline Sapotaceae & Chrysophyllum splendens Spreng. & 1 & 3,3 & 7 & 0,9 & 0,04 & 0,17 & 1 & 4,4 & ARC 200 \\
\hline Myrtaceae & Calyptranthes concinna DC. & 1 & 3,3 & 3 & 0,4 & 0,16 & 0,59 & 1 & 4,4 & ARC 34 \\
\hline Euphorbiaceae & $\begin{array}{l}\text { Pachystroma longifolium (Ness) I.M. } \\
\text { Johns }\end{array}$ & 1 & 3,3 & 7 & 0,9 & 0,03 & 0,11 & 1 & 4,3 & ARC 201 \\
\hline Meliaceae & Trichilia pseudostipularis C.DC. & 1 & 3,3 & 3 & 0,4 & 0,13 & 0,50 & 0,9 & 4,3 & ARC 99 \\
\hline Bignoniaceae & Tabebuia stenocalyx Sprague et Stapf & 1 & 3,3 & 3 & 0,4 & 0,13 & 0,49 & 0,9 & 4,2 & ARC 132 \\
\hline Lauraceae & Nectandra oppositifolia Nees & 1 & 3,3 & 3 & 0,4 & 0,12 & 0,44 & 0,9 & 4,2 & $\mathrm{~s} / \mathrm{n}^{\circ}$ \\
\hline Lauraceae & Ocotea odorifera (Vell.) Rohwer & 1 & 3,3 & 3 & 0,4 & 0,11 & 0,42 & 0,8 & 4,2 & ARC 202 \\
\hline
\end{tabular}




\begin{tabular}{|c|c|c|c|c|c|c|c|c|c|c|}
\hline Família & Nome Científico & $\mathbf{F A}$ & FR & DA & DR & DoA & DoR & $\mathrm{VC}$ & VI & $\begin{array}{c}\mathrm{N}^{\mathbf{0}} \\
\text { coletor }\end{array}$ \\
\hline Moraceae & Ficus cestrifolia Schott & 1 & 3,3 & 3 & 0,4 & 0,11 & 0,40 & 0,8 & 4,2 & ARC 204 \\
\hline Brassicaceae & Crateva tapia $\mathrm{L}$. & 1 & 3,3 & 3 & 0,4 & 0,09 & 0,35 & 0,8 & 4,1 & ARC 205 \\
\hline Euphorbiaceae & Croton celtidifolius Baill. & 1 & 3,3 & 3 & 0,4 & 0,09 & 0,34 & 0,8 & 4,1 & ARC 206 \\
\hline Sapotaceae & Manilkara salzmannii (A.DC.) Lam. & 1 & 3,3 & 3 & 0,4 & 0,08 & 0,29 & 0,7 & 4,1 & ARC 207 \\
\hline Fab. Caesalpinioideae & Schizolobium parahyba (Vell.) S.F. Blake & 1 & 3,3 & 3 & 0,4 & 0,08 & 0,29 & 0,7 & 4,1 & $\mathrm{~s} / \mathrm{n}^{\circ}$ \\
\hline Myrtaceae & Marlierea tomentosa Cambess. & 1 & 3,3 & 3 & 0,4 & 0,07 & 0,26 & 0,7 & 4 & ARC 20 \\
\hline Chrysobalanaceae & Licania kunthiana Hook. F. & 1 & 3,3 & 3 & 0,4 & 0,07 & 0,25 & 0,7 & 4 & ARC 88 \\
\hline Myrtaceae & Campomanesia laurifolia Gardner & 1 & 3,3 & 3 & 0,4 & 0,06 & 0,24 & 0,7 & 4 & $\mathrm{ARC} 12$ \\
\hline Apocynaceae & Aspidosperma ramiflorum Müll. Arg. & 1 & 3,3 & 3 & 0,4 & 0,05 & 0,20 & 0,6 & 4 & ARC 08 \\
\hline Bignoniaceae & Cybistax antisyphilitica (Mart.) Mart. & 1 & 3,3 & 3 & 0,4 & 0,05 & 0,20 & 0,6 & 4 & $\mathrm{ARC} 45$ \\
\hline Elaeocarpaceae & Sloanea guianensis (Aubl.) Benth. & 1 & 3,3 & 3 & 0,4 & 0,04 & 0,17 & 0,6 & 3,9 & ARC 210 \\
\hline Anacardiaceae & Tapirira guianensis Aubl. & 1 & 3,3 & 3 & 0,4 & 0,04 & 0,16 & 0,6 & 3,9 & $\mathrm{~s} / \mathrm{n}^{\circ}$ \\
\hline Urticaceae & Coussapoa microcarpa (Shott) Rizzini & 1 & 3,3 & 3 & 0,4 & 0,03 & 0,13 & 0,6 & 3,9 & ARC 209 \\
\hline Lauraceae & Nectandra puberula (Schott) Nees & 1 & 3,3 & 3 & 0,4 & 0,03 & 0,13 & 0,6 & 3,9 & ARC 179 \\
\hline Celastraceae & Maytenus brasiliensis Mart. & 1 & 3,3 & 3 & 0,4 & 0,03 & 0,11 & 0,5 & 3,9 & $\mathrm{~s} / \mathrm{n}^{\circ}$ \\
\hline Melastomataceae & Miconia prasina (Sw.) DC. & 1 & 3,3 & 3 & 0,4 & 0,03 & 0,10 & 0,5 & 3,9 & $\mathrm{~s} / \mathrm{n}^{\circ}$ \\
\hline Chrysobalanaceae & Licaria armeniaca (Nees) Kosterm. & 1 & 3,3 & 3 & 0,4 & 0,03 & 0,10 & 0,5 & 3,9 & ARC 212 \\
\hline Solanaceae & Solanum granulosoleprosum Dunal & 1 & 3,3 & 3 & 0,4 & 0,03 & 0,10 & 0,5 & 3,9 & ARC 55 \\
\hline Myrtaceae & Myrcia splendens (Sw.) DC. & 1 & 3,3 & 3 & 0,4 & 0,02 & 0,09 & 0,5 & 3,9 & ARC 01 \\
\hline Meliaceae & Cabralea canjerana (Vell.) Mart & 1 & 3,3 & 3 & 0,4 & 0,02 & 0,09 & 0,5 & 3,9 & $\mathrm{~s} / \mathrm{n}^{\circ}$ \\
\hline Fab. Mimosoideae & Inga af. cylindrica (Vell.) Mart. & 1 & 3,3 & 3 & 0,4 & 0,02 & 0,09 & 0,5 & 3,9 & ARC 213 \\
\hline Phyllanthaceae & Hieronyma alchoneoides Allemão & 1 & 3,3 & 3 & 0,4 & 0,02 & 0,07 & 0,5 & 3,8 & ARC 180 \\
\hline Apocynaceae & Rauvolfia grandiflora Mart. ex A.DC. & 1 & 3,3 & 3 & 0,4 & 0,02 & 0,06 & 0,5 & 3,8 & ARC 181 \\
\hline Meliaceae & Trichilia elegans A. Juss & 1 & 3,3 & 3 & 0,4 & 0,02 & 0,06 & 0,5 & 3,8 & ARC 22 \\
\hline Apocynaceae & Geissospermum laeve (Vell.) Miers & 1 & 3,3 & 3 & 0,4 & 0,02 & 0,06 & 0,5 & 3,8 & ARC 61 \\
\hline Fab. Papilionoideae & Machaerium nictitans (Vell.) Benth. & 1 & 3,3 & 3 & 0,4 & 0,02 & 0,06 & 0,5 & 3,8 & ARC 65 \\
\hline Myrtaceae & Myrcia spectabilis DC. & 1 & 3,3 & 3 & 0,4 & 0,01 & 0,05 & 0,5 & 3,8 & $\mathrm{~s} / \mathrm{n}^{\circ}$ \\
\hline Malpighiaceae & Byrsonima sericea DC. & 1 & 3,3 & 3 & 0,4 & 0,01 & 0,05 & 0,5 & 3,8 & ARC 54 \\
\hline Rubiaceae & Faramea includens Müll. Arg. & 1 & 3,3 & 3 & 0,4 & 0,01 & 0,05 & 0,5 & 3,8 & ARC 182 \\
\hline Phytolaccaceae & Gallesia integrifolia (Spreng.) Harms & 1 & 3,3 & 3 & 0,4 & 0,01 & 0,05 & 0,5 & 3,8 & $\mathrm{~s} / \mathrm{n}^{\circ}$ \\
\hline Lauraceae & Ocotea divaricata (Nees) Mez & 1 & 3,3 & 3 & 0,4 & 0,01 & 0,04 & 0,5 & 3,8 & $\mathrm{~s} / \mathrm{n}^{\circ}$ \\
\hline Meliaceae & Trichilia lepidota Mart. & 1 & 3,3 & 3 & 0,4 & 0,01 & 0,04 & 0,5 & 3,8 & ARC 07 \\
\hline Apocynaceae & Tabernaemontana hystrix Steud. & 1 & 3,3 & 3 & 0,4 & 0,01 & 0,04 & 0,5 & 3,8 & ARC 222 \\
\hline \multirow[t]{2}{*}{ Myrtaceae } & Myrcia plusiantha Kiaersk & 1 & 3,3 & 3 & 0,4 & 0,01 & 0,03 & 0,5 & 3,8 & ARC 27 \\
\hline & TOTAIS & & & 780 & 100 & 26,57 & 100 & 200 & 583,2 & \\
\hline
\end{tabular}


amostrar a maior parte riqueza de espécies das duas áreas estudadas, já que o incremento em ambas as áreas nas últimas parcelas foi relativamente pequeno $(\sim 1,5 \%)$ (Fig. 1). Quando avaliada a diversidade de Shannon, o PNMFA apresentou valor intermediário de diversidade $\left(H^{\prime}=3,96\right)$ em relação a outras localidades do estado do Rio de Janeiro (Tab. 1). Apesar da A1 apresentar menor valor de diversidade $\left(3,58\right.$ nats.indivíduo $\left.{ }^{-1}\right)$ que a

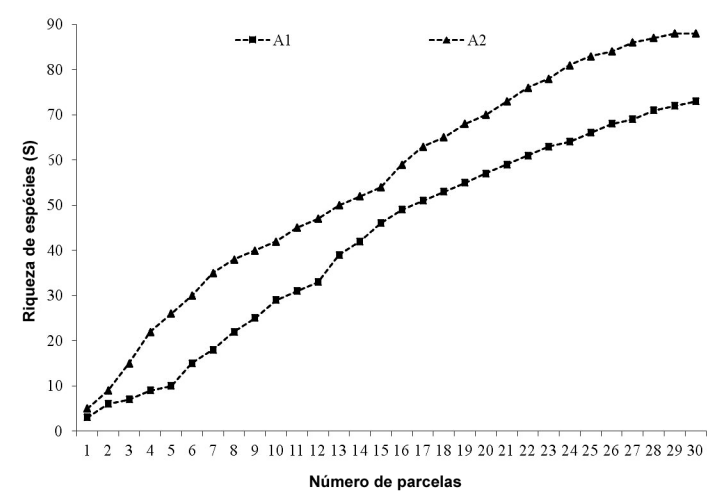

Figura 1 - Curvas do coletor das áreas 1 (A) e 2 (B) analisadas no Parque Natural Municipal Fazenda Atalaia, município de Macaé, Rio de Janeiro.

Figure 1 - Colector's curves of the area 1 (A) and 2 (B) analyzed in the Parque Natural Municipal Fazenda Atalaia, Macaé municipality, Rio de Janeiro.
A2 $(3,94)$, não houve diferença significativa entre os dois valores (teste $\mathrm{t}$ modificado de Hutcheson: $\mathrm{p}>0,05)$.

A similaridade de Jaccard na composição de espécies entre as duas áreas amostradas foi de $36,4 \%$. Já os valores de similaridade obtidos entre o PNMFA e os dos demais estudos podem ser considerados baixos, variando de $8 \%$ (Reserva Biológica de Poço das Antas) a 19\% (Região do Imbaú) (Tab. 5). O diagrama de ordenação gerado pela NMDS não apresentou um padrão claro de agrupamento entre o PNMFA e as demais áreas analisadas (Stress de Kruskal $=0,072$; Proporção da variância $[\mathrm{RSQ}]=0,969$; $\mathrm{R}=0,126 ; \mathrm{p}=0,68)$ (Fig. 2).

$\mathrm{A}$ área basal $(\mathrm{AB})$ obtida para as duas áreas foi de $35,6 \mathrm{~m}^{2} /$ ha (A1) e $26,6 \mathrm{~m}^{2} / \mathrm{ha}(\mathrm{A} 2)$. $\mathrm{Na}$ A1, $53,1 \%$ da DoA se deve a quatro espécies (Artocarpus heterophyllus, Ficus adhatodaefolia, Cybistax antisyphilitica e Astronium fraxifolium) (Tab. 3). Já na A2, as espécies Ficus gomelleira, A. heterophyllus, Cedrella fissilis e Croton floribundus corresponderam a 50,2\% da DoA (Tab. 4).

Artocarpus heterophyllus foi a espécie que apresentou os maiores valores de importância e de cobertura (Tabs. 3 e 4) nas duas áreas analisadas. $\mathrm{Na}$ A1, Guapira opposita e Senefeldera verticilata foram as espécies que apresentaram o segundo e o terceiro valores mais altos desses parâmetros,

Tabela 5 - Matriz de similaridade - índice de similaridade de Jaccard (Cj\%). PNMFA = Parque Natural Municipal Fazenda Atalaia; EEEP = Estação Ecológica Estadual do Paraíso; MC = Mata do Carvão; IMBE = Região do Imbé; IMBAU = Região do Imbaú; MRV = Mata Rio Vermelho; RBPA = Reserva Biológica de Poço das Antas; MBJ = Mata do Bom Jesus; FB = Fazenda Biovert; RBU = Reserva Biológica União.

Table 5 - Values of Jaccard similarity index $(\mathrm{Cj} \%)$ obtained for the locations analyzed in the study. PNMFA = Parque Natural Municipal Fazenda Atalaia; EEEP = Estação Ecológica Estadual do Paraíso; $\mathrm{MC}=$ Mata do Carvão; IMBE = Região do Imbé; IMBAU = Região do Imbaú; MRV = Mata Rio Vermelho; RBPA = Reserva Biológica de Poço das Antas; MBJ = Mata do Bom Jesus; FB=Fazenda Biovert; RBU = Reserva Biológica União.

\begin{tabular}{lccccccccc}
\hline & PNMFA & EEEP & MC & IMBE & IMBAU & MRV & RBPA & MBJ & FB \\
\hline EEEP & 0,14 & 1 & - & - & - & - & - & - & - \\
MC & 0,10 & 0,03 & 1 & - & - & - & - & - & - \\
IMBE & 0,17 & 0,21 & 0,05 & 1 & - & - & - & - & - \\
IMBAU & 0,19 & 0,08 & 0,09 & 0,15 & 1 & - & - & - & - \\
MRV & 0,15 & 0,08 & 0,07 & 0,13 & 0,40 & 1 & - & - & - \\
RBPA & 0,08 & 0,07 & 0,05 & 0,11 & 0,15 & 0,14 & 1 & - & - \\
MBJ & 0,13 & 0,07 & 0,23 & 0,08 & 0,19 & 0,17 & 0,11 & 1 & - \\
FB & 0,12 & 0,11 & 0,05 & 0,15 & 0,21 & 0,20 & 0,09 & 0,09 & 1 \\
RBU & 0,13 & 0,10 & 0,04 & 0,22 & 0,20 & 0,18 & 0,13 & 0,09 & 0,15 \\
\hline
\end{tabular}




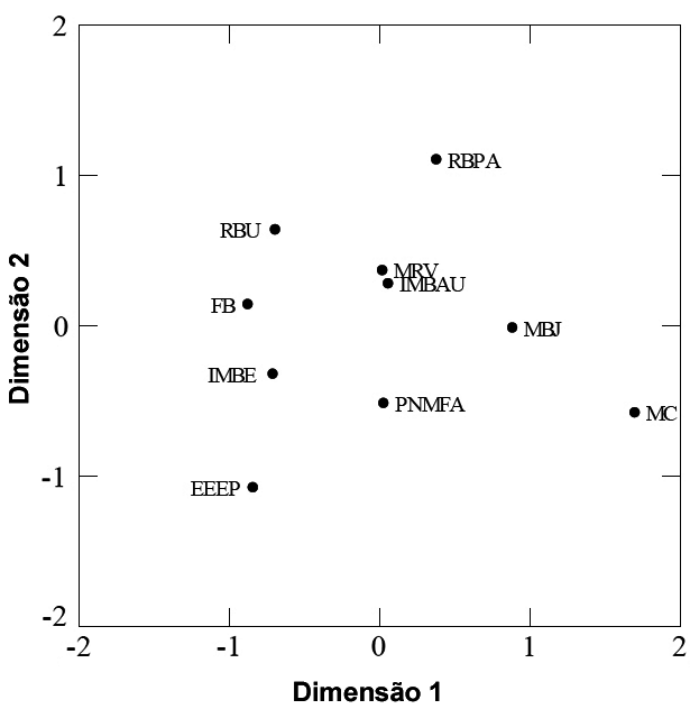

Figura 2 - Diagrama de ordenação obtido por escalonamento multidimensional não-métrico (NMDS) para o Parque Natural Fazenda Atalaia e para outras áreas de Floresta Atlântica do Estado do Rio de Janeiro. Stress $=0,073$; Proporção da variância $(\mathrm{RSQ})=0,969$. PNMFA = Parque Natural Municipal Fazenda Atalaia; EEEP = Estação Ecológica Estadual do Paraíso; $\mathrm{MC}=$ Mata do Carvão; IMBE = Região do Imbé; IMBAU = Região do Imbaú; MRV = Mata Rio Vermelho; RBPA = Reserva Biológica de Poço das Antas; MBJ = Mata do Bom Jesus; $\mathrm{FB}=$ Fazenda Biovert; RBU $=$ Reserva Biológica União. Figure 2 - Ordination diagram obtained by the ordination method of non-metric muldimensional scaling (NMDS) for the Parque Natural Fazenda Atalaia and other areas of Atlantic forest of Rio de Janeiro state. Kruskal Stress = 0,073; Variance proportion $(R S Q)=0,969$. PNMFA $=$ Parque Natural Municipal Fazenda Atalaia; EEEP = Estação Ecológica Estadual do Paraíso; $\mathrm{MC}=$ Mata do Carvão; IMBE = Região do Imbé; IMBAU = Região do Imbaú; MRV = Mata Rio Vermelho; RBPA = Reserva Biológica de Poço das Antas; MBJ = Mata do Bom Jesus; FB = Fazenda Biovert; RBU = Reserva Biológica União.

respectivamente (Tab. 3), enquanto em A2 essas colocações foram ocupadas por F. gomelleira e $C$. floribundus (Tab. 4).

\section{Discussão}

Fabaceae, Euphorbiaceae, Myrtaceae, Meliaceae e Sapotaceae são as famílias mais representativas em termos de número de espécies no PNMFA e na maior parte dos estudos em áreas do Norte do estado (Kurtz \& Araújo 2000; Silva \& Nascimento 2001; Moreno et al. 2003; GuedesBruni et al. 2006; Carvalho et al. 2006a; Carvalho et al. 2007). Segundo Oliveira-Filho \& Fontes (2000), essas famílias são típicas da Floresta Ombrófila Densa de Terras Baixas do Sudeste brasileiro. Outras famílias, tais como Euphorbiaceae e Myrtaceae, também representativas no PNMFA, são típicas das matas de tabuleiro e de terras baixas (Silva \& Nascimento 2001).

Na maioria das áreas comparadas, os valores de riqueza são superiores aos obtidos para PNMFA (Borém \& Oliveira-Filho 2002; Moreno et al. 2003; Rodrigues 2004; Carvalho et al. 2006a; GuedesBruni et al. 2006). O valor registrado para a área estudada pode ser um efeito de fatores relacionados ao tempo de regeneração natural das áreas estudadas no PNMFA, que sofreu seu último manejo (corte seletivo) há cerca de 50 anos. A partir de então, a área foi abandonada e vem sofrendo recolonização, não somente por espécies de estágios sucessionais iniciais (e.g. Cecropia pachystachya), mas também por aquelas de estágios mais avançados (e.g. Apuleia leiocarpa e Brosimum guianensis).

Os valores de diversidade (H') obtidos para o PNMFA como um todo e para cada um dos trechos analisados podem ser considerados de baixos a medianos quando comparados aos de outras áreas de mata do estado, como as da Reserva Biológica União ( $\mathrm{H}^{\prime}=4,90$ nats.indivíduo ${ }^{-1}$; Rodrigues 2004), da Reserva Biológica de Poço das Antas $\left(\mathrm{H}^{\prime}=4,57\right.$ nats.indivíduo ${ }^{-1}$; Guedes-Bruni et al. 2006) e da Estação Ecológica Estadual do Paraíso ( $\mathrm{H}^{\prime}=4,20$ nats.indivíduo ${ }^{-1}$; Kurtz \& Araújo 2000).

Os baixos valores de similaridade obtidos para o PNMFA e os outros estudos realizados na região Centro-Norte do estado e a ausência de agrupamentos claros na análise de ordenação (NMDS) sugerem que as áreas analisadas apresentam composição florística bastante distintas, com grupos de espécies peculiares a cada uma das áreas. Esses baixos valores de similaridade podem ser explicados por fatores relacionados às características geoclimáticas das áreas estudadas (altitude, temperatura e pluviosidade) (Gentry 1982; Oliveira-Filho \& Fontes 2000; Nettesheim et al. 2010) e pelo histórico de degradação sofrido (Carvalho et al. 2006b). Apesar das áreas estarem localizadas na região Centro-Norte do estado do Rio de Janeiro, com algumas estando geograficamente próximas entre si (p. ex., as REBIOs Poço das Antas e União), elas apresentam diferenças na altitude e orientação geográfica, além de estarem submetidas a diferentes regimes pluviométricos, que são fatores importantes para determinar a 
ocorrência de espécies arbóreas (Gentry 1982; Oliveira-Filho \& Fontes 2000; Nettesheim et al. 2010), especialmente em ambientes com elevada heterogeneidade ambiental e climática como a Floresta Atlântica do Rio de Janeiro (Peixoto et al. 2004; Carvalho et al. 2006b; Rolim et al. 2006). Além disso, é possível que o histórico de degradação e o tempo de regeneração das áreas analisadas exerçam efeito sobre o atual estágio de regeneração das mesmas (Clark 1996; Oliveira 2002). No entanto, seriam necessários estudos mais aprofundados sobre esses históricos nas áreas analisadas de forma a tornar essa afirmação mais consistente.

Em relação a outros estudos, o valor de similaridade entre as duas áreas analisadas (A1 e A2) pode ser considerado elevado (MuellerDombois \& Ellenberg 1974), o que pode ser uma resposta da pequena distância linear entre os dois trechos de vegetação (cerca de $1 \mathrm{~km}$ ) e ao fato de estarem no mesmo fragmento. Essa distância relativamente curta, associada à ausência de barreiras físicas, permitiria a dispersão de propágulos entre as duas áreas, tanto abiótica quanto bioticamente (Alcantara et al. 2000; Dalling et al. 2002; Levine \& Murrell 2003). Isso permitiria a manutenção de um grande número de espécies comuns e, consequentemente, explicaria a elevada similaridade entre as duas áreas.

Por outro lado, as diferenças encontradas entre as áreas podem ser explicadas, principalmente, por estarem em vertentes distintas de um mesmo vale, já que a A1 estava voltada para o Norte e A2 para o Sul. Diversos estudos têm demonstrado que as vertentes têm um efeito direto sobre a composição de espécies em uma mesma localidade (Moustafa \& Zayed 1996; Guerrero-Campo et al. 1999; Kinupp \& Magnusson 2005), já que as condições microclimáticas, tais como aporte de umidade, grau e duração da insolação, podem ter um efeito sobre a abundância, riqueza e estrutura da vegetação (Tuosmito et al. 1995; Clark et al. 1998; Small \& McCarthy 2002).

Nas duas áreas analisadas, a espécie que apresentou os maiores valores de dominância foi Artocarpus heterophyllus (jaqueira), uma espécie exótica que foi introduzida nessa região para fins horticulturais, como em todo o Brasil (Chaves et al. 1967). As populações desta espécie podem apresentar de três a quatro eventos reprodutivos por ano, com um indivíduo sadio podendo produzir até 100 frutos por ano (Chaves et al. 1967).
Além disso, A. heterophyllus parece não possuir herbívoros e predadores capazes de regular suas populações nas áreas de Mata Atlântica em que foi introduzida (hipótese do escape dos inimigos; Sax \& Brown 2000; Keane \& Crawley 2002); muitas espécies de mamíferos nativos (tais como cutias, gambás e primatas) têm sido registradas como potenciais dispersoras de suas sementes (Cunha et al. 2006), que possuem elevadas taxas de germinação, especialmente em áreas de borda (Abreu \& Rodrigues 2010). Esse conjunto de características faz com que esta espécie apresente um extremo potencial invasor e, consequentemente, de modificação da flora nativa dessas áreas, como em outras unidades de conservação do estado do Rio de Janeiro, tais como o Parque Nacional da Tijuca e as Reservas Biológicas de Poço das Antas, da União e do Tinguá (Abreu \& Rodrigues 2010). Dessa forma, é necessário que no PNMFA sejam estabelecidos programas de manejo e controle dessa espécie, evitando, assim, a modificação da biota local e a possível extinção de algumas espécies mais sensíveis à competição com A. heterophyllus.

A maior parte das espécies do PNMFA pode ser considerada como tendendo à raridade (sensu Martins 1993; Kurtz \& Araujo 2000), já que apresentam valores muito baixos de abundância, frequência de ocorrência e densidade relativa, em geral sendo representados por menos de dois indivíduos $(\mathrm{Dr}=0,5)$ (Tab. 3 e 4). Assim, 38,4\% das espécies $(\mathrm{N}=28$ espécies $)$ e $76,1 \%(\mathrm{~N}=$ 67) foram representadas respectivamente na $\mathrm{A} 1$ e A2 por densidades relativas menores que 0,5 indivíduo. O número de espécies consideradas raras neste estudo pode ser considerado elevado, já que tem sido sugerido que para a Floresta Atlântica do Estado Rio de Janeiro os valores de raridade oscilam entre 9,5 e 45,2\% (Kurtz \& Araújo 2000). No entanto, esta afirmativa não deve ser aceita como um padrão, já que esses valores podem ser influenciados pelo esforço amostral, pelo critério de inclusão e pela organização espacial das amostras (Kurtz \& Araújo 2000).

Os valores de área basal para as duas áreas analisadas do PNMFA podem ser considerados de intermediários a elevados quando comparados com outras localidades com o mesmo tipo de floresta (Tab. 1). Esse resultado pode refletir o grau de perturbação antrópica que as duas áreas sofreram no passado, como o corte seletivo de madeira, retirada de lenha, prováveis queimadas e o estágio 
de regeneração da área, estando cada uma delas em momento distinto da sucessão ecológica. Segundo moradores de fazendas próximas ao PNMFA, há cerca de 50 anos a área não sofre corte de árvores (A.F. Nunes Freitas, obs. pess.), o que permitiria que as espécies realizassem seu crescimento e reprodução sem efeito de ação antrópica direta e apresentando ainda os baixos valores de área basal. Este fato pode ser um indicativo de que a maioria das espécies esteja recolonizando a área, mas ainda são necessários estudos mais aprofundados sobre as taxas de recuperação das populações das diferentes espécies, tais como estudos sobre chuva e banco de sementes, e sobre o recrutamento das espécies nativas.

Os resultados deste estudo sugerem que o Parque Natural Municipal Fazenda Atalaia, apesar dos distúrbios sofridos em um passado recente, ainda mantém uma parcela da diversidade arbórea da região norte-fluminense. Boa parte das espécies ainda apresenta baixas densidades e está em estágio inicial de ocupação do PNMFA. No entanto, medidas de manejo, controle e erradicação de algumas espécies, em especial da exótica invasora A. heterophyllus, são essenciais para impedir que esta exclua outras espécies e inviabilize o processo de regeneração natural de espécies nativas da região.

\section{Agradecimentos}

À Secretaria Municipal de Meio Ambiente do Município de Macaé, a autorização para realizar o estudo no Parque Natural Municipal Fazenda Atalaia e à A R.D. Antonini e T.C. Rocha-Pessôa, as importantes contribuições na leitura do manuscrito. Este estudo fez parte do Projeto de Pesquisa Estrutura e dinâmica de comunidades vegetais em fragmentos de Mata Atlântica do Rio de Janeiro e foi parcialmente subvencionado com Auxílio Instalação da Fundação Carlos Chagas Filho de Amparo à Pesquisa do Estado do Rio de Janeiro FAPERJ (Processo No E-26/170.238/2006).

\section{Referências}

Abreu, R.C.R. \& Rodrigues, P.J.F.P. 2010. Exotic tree Artocarpus heterophyllus (Moraceae) invades Brazilian Atlantic Forest. Rodriguésia 61: 677-688.

Alcantara, J.M.; Rey, P.J. \& Sanchez-Lafuente, A.M. 2000. Factors shaping the seedfall pattern of a birddispersed plant. Ecology 81: 1937-1950.

APG III. 2009. An update of the Angiosperm Phylogeny Group classification for the orders and families of flowering plants: APG III. Botanical Journal of the Linnean Society 161: 105-121.

Borém, R.A.T. \& Oliveira-Filho, A.T. 2002. Fitossociologia do estrato arbóreo em uma toposseqüência alterada de mata Atlântica, no município de Silva Jardim-RJ, Brasil. Revista Árvore 26: 727-742.

Carvalho, F.A. 2001. Estrutura e fitossociologia da borda e do interior de dois fragmentos de Mata Atlântica de baixada periodicamente alagados na REBIO Poço das Antas, RJ. Monografia de Bacharelado. Universidade Estadual do Norte Fluminense, Campos dos Goytacazes. 67p.

Carvalho, F.A.; Braga, J.M.A.; Gomes, J.M.L.; Souza, J.S. \& Nascimento, M.T. 2006a. Comunidade arbórea de uma floresta de baixada aluvial no município de Campos dos Goytacazes, RJ. CERNE 12: 157-166.

Carvalho, F.A.; Nascimento, M.T. \& Braga, J.M.A. 2006 b. Composição e riqueza florística do componente arbóreo da Floresta Atlântica submontana na região de Imbaú, Município de Silva Jardim, RJ. Acta Botanica Brasilica 20: 727-740.

Carvalho, F.A.; Nascimento, M.T. \& Braga, J.M.A. 2007. Estrutura e composição florística do estrato arbóreo de um remanescente de Mata Atlântica submontana no município de Rio Bonito, RJ, Brasil (Mata Rio Vermelho). Revista Árvore 31: 717-730.

Chaves, C.M.; Martins, H.F.; Carauta, J.P.P.; LannaSobrinho, J.P.; Vianna, M.C. \& Silva, S.A.F. 1967. Arboreto carioca 3. Centro de Conservação da Natureza, Rio de Janeiro. 28p.

Clark, D.B. 1996. Abolishing virginity. Journal of Tropical Ecology 12: 435-439.

Clark, D.B.; Clark, D.A. \& Read, J.M. 1998. Edaphic variation and the mesoescale distribution of tree species in a neotropical rain forest. Journal of Ecology 86: 10-112.

Cowell, R.K. 2006. EstimateS: statistical estimation of species richness and shared species from samples. Version 8.

Cunha, A.A.; Vieira, M.V. \& Grelle, C.E.V. 2006. Preliminary observations on habitat, support use and diet in two non-native primates in an urban Atlantic forest fragment: The capuchin monkey (Cebus sp.) and the common marmoset (Callithrix jacchus) in the Tijuca forest, Rio de Janeiro. Urban Ecosystems 9: 351-359.

Dalling, J.; Muller-Landau, H.C.; Wright, J. \& Hubbell, S.P. 2002. Role of dispersal in the recruitment limitation of neotropical pioneer tress. Journal of Ecology 90: 714-727.

Eisenlohr, P.V.; Melo, M.M.R.F.; Ivanauskas, N.M.; Souza, V.C.; Rodrigues, R.R.; Duarte, A.R.; Breier, T.B.; Udulutsch, R.G. 2011. Floresta Ombrófila Densa Atlântica: bases conceituais e estudo de caso no Parque Estadual de Carlos Botelho, estado de 
São Paulo. In: Felfili, J.M.; Eisenlohr, P.V.; Melo, M.M.R.F.; Andrade, L.A.; Meira Neto, J.A.A. (orgs.). Fitossociologia no Brasil: métodos e estudos de casos. UFV, Viçosa. Pp. 372-387.

Fundação SOS Mata Atlântica \& INPE. 2001. Atlas dos Remanescentes Florestais da Mata Atlântica - Período 1995-2000 - Relatório Parcial - Estado do Rio de Janeiro. Fundação SOS Mata Atlântica e Instituto Nacional de Pesquisas Espaciais, São Paulo. 47p.

Fundação SOS Mata Atlântica \& INPE. 2010. Atlas dos Remanescentes Florestais da Mata Atlântica - Período 2008-2010 - Relatório Parcial - Estado do Rio de Janeiro. Fundação SOS Mata Atlântica e Instituto Nacional de Pesquisas Espaciais, São Paulo. 60p.

Gentry, A.H. 1982. Patterns of Neotropical Plant Species Diversity. Evolutionary Biology 15: 1-84.

Guatura, I.N.; Corrêa, F.; Costa, J.P.O. \& Azevedo, P.U.E. 1996. A questão fundiária: roteiro para a solução dos problemas fundiários nas áreas protegidas da Mata Atlântica. Roteiro para a conservação de sua biodiversidade. Série Conservação e Áreas Protegidas 1. $2^{\mathrm{a}}$ ed. Conselho Nacional da Reserva da Biosfera da Mata Atlântica, São Paulo. 47p.

Guedes-Bruni, R.R.; Neto S.J.S.; Morim, M.P. \& Mantovani, W. 2006. Composição Florística e Estrutura de Dossel em Trecho de Floresta Ombrófila Densa Atlântica sobre Morrote Mamelonar na Reserva Biológica de Poços das Antas, Silva Jardim, Rio de Janeiro, Brasil. Rodriguésia 57: 429-442.

Guerrero-Campo, J.; Alberto, F.; Hodgson, J.; GarcíaRuiz, J.M. \& Montserrat-Martí, G. 1999. Plant community patterns in a gypsum area of NE Spain. I. Interactions with topographic factors and soil erosion. Journal of Arid Environments 41: 401-410.

Keane, R.M. \& Crawley, M.J. 2002. Exotic plant invasions and the enemy release hypothesis. Trens in Ecology \& Evolution 17:164-170.

Kinupp, V.F. \& Magnusson, W.E. 2005. Spatial patterns in the understory shrub genus Psychotria in central Amazonia: effects of distance and topography. Journal of Tropical Ecology 21: 363-374.

Kurtz, B.C.\& Araújo, D.S.D. 2000. Composição florística e estrutura do componente arbóreo de um trecho de Mata Atlântica na Estação Ecológica do Paraíso, Cachoeiras do Macacú, RJ, Brasil. Rodriguésia 51: 69-112.

Levine, J.M. \& Murrell, D.J. 2003. The community-level consequences of seed dispersal patterns. Annual Review of Ecology, Evolution and Systematic 34: 549-574.

Legendre, P. \& Legendre, L. 1998. Numerical ecology. Elsevier Science B.V. 853p.

Magurran, A.E. 2004. Measuring biological diversity. Blackwell Publishing, Oxford. 179p.

Martins, F.R. 1993. Estrutura de uma floresta mesófila. $2^{\text {a }}$ ed. Editora da UNICAMP, Campinas. 256p.
Morellato, L.P.C \& Haddad, C.F.B. 2000. Introduction: the Brazilian Atlantic Forest. Biotropica 32: 786-792.

Moreno, M.R.; Nascimento, M.T. \& Kurtz, B.C. 2003. Estrutura e composição florística do estrato arbóreo em duas zonas altitudinais na Mata Atlântica de encosta da região do Imbé, RJ. Acta Botanica Brasilica 17: 371-386.

Moustafa, A.E-R.A. \& Zayed, A. 1996. Effects of environmental factors on the flora of alluvial fans in southern Sinai. Journal of Arid Environments 32: 431-443.

Mueller-Dumbois, D. \& Ellenberg, H. 1974. Aims and methods of vegetation ecology. John Wiley, New York. 547p.

Nettesheim, F.C.;Menezes, L.F.T.; Carvalho, D.C.; Conde, M.M.S. \& Araújo, D.S.D. 2010. Influence of environmental variation on Atlantic Forest treeshrub-layer phytogeography in southeast Brazil. Acta Botanica Brasilica 24: 369-377.

Oliveira, R.R. 2002. Ação antrópica e resultantes sobre a estrutura e composição da Mata Atlântica na Ilha Grande, RJ. Rodriguésia 53: 33-58.

Oliveira-Filho, A.T. \& Fontes, M.A. 2000. Patterns of floristic differentiation among Atlantic Forest in Southeastern Brazil and the influence of climate. Biotropica 32: 793809.

Pagano, S.N.; Leitão-Filho, H.F. \& Shepherd, G.J. 1987. Estudo fitossociológico em Mata Mesófila semidecídua no município de Rio Claro (estado de São Paulo). Revista Brasileira de Botânica 10: 49-62.

Peixoto, G.L.; Martins, S.V.; Silva, A.F. \& Silva, E. 2004. Composição floristica do componente arbóreo de um trecho de Floresta Atlântica na Área de Proteção Ambiental da Serra da Capoeira Grande, Rio de Janeiro, RJ, Brasil. Acta Botanica Brasilica 18: 151-160.

Peres, W.R. \& Rahy, I.S. 2001. Índice de Qualidade dos Municípios Verde (IQM-Verde) - Instrumento para o planejamento ecológico e gestão ambiental do território do Estado do Rio de Janeiro. In: Anais do X SBSR. INPE, Foz do Iguaçu. Pp. 1147-1154.

Pillar, V.D. 2004. Suficiência amostral. In: C.E.M. Bicudo \& D.C. Bicudi (eds.). Amostragem em Limnologia. Rima, São Carlos. Pp. 25-43.

Rocha, C.F.D.; Bergallo, H.G.; Alves, M.A.S. \& Van Sluys, M. 2003. A biodiversidade nos grandes remanescentes florestais do estado do Rio de Janeiro e nas restingas da Mata Atlântica. Editora RiMa, São Carlos. 160p.

Rodrigues, P.J.F.P. 2004. A vegetação da Reserva Biológica União e os efeitos de borda na Mata Atlântica. Tese de Doutorado. Universidade Estadual do Norte Fluminense, Campos dos Goytacazes. 136p.

Rolim, S.G.; Ivanauskas, N.M.; Rodrigues, R.R.; Nascimento, M.T.; Gomes, J.M.L.; Folli, D.A. \& Couto, H.T.Z. 2006. Composição florística do estrato arbóreo da Floresta Estacional Semidecidual 
na Planície Aluvial do rio Doce, Linhares, ES, Brasil. Acta Botanica Brasilica 20: 549-561.

Sax, D.F. \& Brown, J.H. 2000. The Paradox of Invasion. Global Ecology \&. Biogeography 9:363-372.

Silva, G.C. \& Nascimento, M.T. 2001. Fitossociologia de um remanescente de mata sobre tabuleiros no norte do estado do Rio de Janeiro (Mata do Carvão). Revista Brasileira de Botânica 24: 51-62.

SIMERJ. 1990. Sistema de Meteorologia do Estado do Rio de Janeiro. Disponível em $<$ http://www.simerj. com>. Acesso em 1 Ago 2007

Small, C.J. \& McCarthy, B.C. 2002. Spatial and temporal variability of herbaceous vegetation in an eastern deciduous forest. Plant Ecology 164: 37-48.

Stehmann, J.R.; Forzza, R.C.; Salino, A.; Sobral, M.; Costa, D.P. \& Kamino, L.H.Y. 2009. Plantas da Floresta Atlântica. 516p.
Tuosmito, H.; Ruokolainen, K.; Kalliola, R.; Linna, A.; Danjoy, W. \& Rodriguez, Z. 1995. Dissecting Amazonian biodiversity. Science 269: 63-66.

Veloso, H.P.; Rangel Filho, A.L.R. \& Lima, J.C.A. 1991. Classificação da vegetação brasileira, adaptada a um sistema universal. Instituto Brasileira de Geografia e Estatística, Departamento de Recursos Naturais e Estudos Ambientais, Rio de Janeiro. $124 \mathrm{p}$.

Vuono, Y.S. 2002. Inventário fitossociológico. In: Sylvestre, L.S. \& Rosa, M.M.T. Manual metodológico para estudos da Mata Atlânica. Editora da Universidade Rural, Seropédica. Pp 51-65.

Wilkinson, L. 1990. SYSTAT: The system for statistics. SYSTAT, Inc., Evanston. 822p.

Zar, J.H. 1999. Biostatistical analysis. $4^{\text {th }}$ ed. Prentice Hall, New Jersey. 663p. 